\title{
Metric properties of alternating Oppenheim expansions
}

by

\author{
János Galambos (Philadelphia, PA), Imre Kátai (Budapest) \\ and Min-Young LeE (Seoul)
}

1. Introduction. Let $a_{n}(j)$ and $b_{n}(j), n \geq 1$, be two sequences of positive integer-valued functions of the positive integers $j \geq 1$, and set

$$
h_{n}(j)=\frac{a_{n}(j)}{b_{n}(j)} j(j-1), \quad j \geq 2 .
$$

Assume that $h_{n}(j)$ is integer-valued $(n \geq 1, j \geq 2)$.

The algorithm $0<x \leq 1, x=x_{1}$, and, for $n \geq 1$, with positive integers $d_{n}$

$$
\frac{1}{d_{n}}<x_{n} \leq \frac{1}{d_{n}-1}, \quad x_{n+1}=\left(x_{n}-\frac{1}{d_{n}}\right) \frac{b_{n}\left(d_{n}\right)}{a_{n}\left(d_{n}\right)}
$$

leads to the series representation

$$
x=\frac{1}{d_{1}}+\frac{a_{1}\left(d_{1}\right)}{b_{1}\left(d_{1}\right)} \frac{1}{d_{2}}+\ldots+\frac{a_{1}\left(d_{1}\right) \ldots a_{n}\left(d_{n}\right)}{b_{1}\left(d_{1}\right) \ldots b_{n}\left(d_{n}\right)} \frac{1}{d_{n+1}}+\ldots
$$

The algorithm (2) implies

$$
d_{n+1}>h_{n}\left(d_{n}\right)
$$

which in turn yields $d_{n} \geq 2$ for each $n \geq 1$. The algorithm (2) never terminates, and (3) with (4) is equivalent to (2). The representation (3) under (4) is unique.

The representation (3) under (2) or (4) was first studied by Oppenheim [8] who established the arithmetical properties, including the question of rationality, of the expansion (2)-(4). Oppenheim's work was first distributed in the form of lecture notes. These notes were used by Galambos [1] where the foundations of the metric theory of (2)-(4) are laid down. Further development, with several new results, can be found in a monograph of Galambos [2]. The expansion (2)-(4) became known as Oppenheim expansion.

2000 Mathematics Subject Classification: Primary 11K55; Secondary 11A67. 
The terms in (3) are all positive. A modification of (2) to the algorithm $0<x \leq 1, x=x_{1}$, and

$$
\begin{aligned}
\frac{1}{D_{n}+1}<x_{n} \leq \frac{1}{D_{n}}, \quad x_{n+1}=\left(\frac{1}{D_{n}}-x_{n}\right) \frac{b_{n}\left(D_{n}\right)}{a_{n}\left(D_{n}\right)}, \\
D_{n} \text { integer, } n \geq 1,
\end{aligned}
$$

generates an alternating series representation. Indeed, if we rearrange the recursive formula in (5) to

$$
x_{n}=\frac{1}{D_{n}}-\frac{a_{n}\left(D_{n}\right)}{b_{n}\left(D_{n}\right)} x_{n+1}
$$

we get

$$
x=\frac{1}{D_{1}}-\frac{a_{1}\left(D_{1}\right)}{b_{1}\left(D_{1}\right)} \frac{1}{D_{2}}+\ldots+(-1)^{n} \frac{a_{1}\left(D_{1}\right) \ldots a_{n}\left(D_{n}\right)}{b_{1}\left(D_{1}\right) \ldots b_{n}\left(D_{n}\right)} x_{n+1} .
$$

The algorithm at (5) terminates for rational $x$ but never for irrationals, that is, (7) always extends to an infinite sum for irrational $x$. In (5) we get

$$
x_{n+1}<\left(\frac{1}{D_{n}}-\frac{1}{D_{n}+1}\right) \frac{b_{n}\left(D_{n}\right)}{a_{n}\left(D_{n}\right)}=\frac{1}{H_{n}\left(D_{n}\right)},
$$

say, yielding

$$
D_{n+1} \geq H_{n}\left(D_{n}\right), \quad n \geq 1,
$$

where

$$
H_{n}(j)=\frac{a_{n}(j)}{b_{n}(j)} j(j+1), \quad j \geq 1 .
$$

We once again assume that $H_{n}(j)$ is integer-valued for $n \geq 1, j \geq 1$.

Note the difference between (1) and (10). Even though (9) implies a faster growth for the sequence $D_{n}=D_{n}(x)$ that what is already known for $d_{n}=d_{n}(x)$ at (2) and (4), we shall establish a remarkable similarity between the metric properties of the two sequences $d_{n}$ and $D_{n}$, valid for almost all $x$ (Lebesgue measure). As usual for metric theory, we shall use Lebesgue measure on the Borel subsets $A$ of the interval $(0,1]$, and write $P(A)$ for this measure.

Very little is known about the metric theory of the sequence $D_{n}$ for general sequences $a_{n}(j)$ and $b_{n}(j)$. In fact, only the special cases $a_{n}(j)=1$ and $b_{n}(j)=j(j+1)$ for all $n \geq 1$ and $j \geq 1$ (the Lüroth case; see Kalpazidou et al. [6] and Indlekofer et al. [5]), and $a_{n}(j)=1$ and $b_{n}(j)=j$ (alternating Engel series or Pierce expansions; see Shallit [9]). We now fill in this gap. For the arithmetical properties of the sequence $D_{n}$, see Indlekofer et al. [5] and their references. 
2. The basic distributional properties. We assume that $x$ is irrational, so the algorithm at (5) does not terminate. It is immediate from (5) that the set of $x$ for which $D_{1}=u, u \geq 1$, is an interval of length $1 /(u(u+1))$, and the set of those $x$ 's for which $D_{t}=u_{t}, 1 \leq t \leq s$, is once again an interval, whose length, by (7), equals

$$
\frac{a_{1}\left(u_{1}\right) \ldots a_{s-1}\left(u_{s-1}\right)}{b_{1}\left(u_{1}\right) \ldots b_{s-1}\left(u_{s-1}\right)} \frac{1}{u_{s}\left(u_{s}+1\right)}
$$

where we assumed that the $u_{t}, 1 \leq t \leq s$, are in conformity with (9). We record these simple observations as

Theorem 1. The sequence $D_{n}, n \geq 1$, satisfies $P\left(D_{1}=u\right)=1 /(u(u+1))$ for $u \geq 1$, and, for $u_{t}, 1 \leq t \leq s, s \geq 2$,

$$
P\left(D_{1}=u_{1}, \ldots, D_{s}=u_{s}\right)=\frac{a_{1}\left(u_{1}\right) \ldots a_{s-1}\left(u_{s-1}\right)}{b_{1}\left(u_{1}\right) \ldots b_{s-1}\left(u_{s-1}\right)} \frac{1}{u_{s}\left(u_{s}+1\right)}
$$

whenever any two consecutive $u_{t}$ satisfy (9). Consequently, the sequence $D_{n}$, $n \geq 1$, forms a Markov chain with transition probabilities

$$
P\left(D_{n+1}=u \mid D_{n}=v\right)=\frac{H_{n}(v)}{u(u+1)}, \quad u \geq H_{n}(v) .
$$

Only (12) needs proof. By definition

$$
\begin{aligned}
P\left(D_{n+1}=u \mid\right. & \left.D_{j}=u_{j}, 1 \leq j \leq n-1, D_{n}=v\right) \\
& =\frac{P\left(D_{1}=u_{1}, \ldots, D_{n-1}=u_{n-1}, D_{n}=v, D_{n+1}=u\right)}{P\left(D_{1}=u_{1}, \ldots, D_{n-1}=u_{n-1}, D_{n}=v\right)} .
\end{aligned}
$$

By (11), the right hand side above does reduce to the right hand side of (12). Since this latter form depends only on $u$ and $v$, both the Markovian property and (12) follow.

$$
\text { Put }
$$

$$
y_{n+1}=H_{n}\left(D_{n}\right) x_{n+1} .
$$

Just as in the case of Oppenheim expansions with positive terms, we have

TheOREM 2. For $n \geq 2, y_{n}$ is uniformly distributed on the interval $(0,1]$, and $y_{n}$ is stochastically independent of the vector $\left(D_{1}, \ldots, D_{n-1}\right)$.

Proof. Let $0<c \leq 1$ be an arbitrary real number. We have to prove

$$
\begin{aligned}
P\left(D_{1}=u_{1}, \ldots, D_{n-1}=u_{n-1}, y_{n} \leq c\right) & \\
& =c P\left(D_{1}=u_{1}, \ldots, D_{n-1}=u_{n-1}\right),
\end{aligned}
$$

where the $u_{t}$ are integers satisfying (9). We once again refer to (7) and (5) and note that the set of those $x$ 's which appear on either side of (14) inside $P()$ is an interval and the length of the interval on the left hand side is exactly $c$ times the length of the one on the right hand side (see also (11)). 
This completes the independence part of the theorem. Upon summing over all possible values of the $u_{t}$ in $(14)$, we now obtain $P\left(y_{n} \leq c\right)=c$. The proof is complete.

TheOREM 2a. Let each $c_{j}$ be the reciprocal of an integer $\geq 2$. Then the events $\left\{y_{j} \leq c_{j}\right\}, 1 \leq j \leq n$, are independent with $P\left(y_{j} \leq c_{j}\right)=c_{j}$.

Proof. Decompose $P\left(y_{1} \leq c_{1}, y_{2} \leq c_{2}, \ldots, y_{n} \leq c_{n}\right)$ as the sum of terms appearing in (11). Upon observing that $y_{j} \leq 1 / k_{j}$, with $k_{j} \geq 2$ integer, is equivalent to $D_{j} \geq k_{j} H_{j-1}\left(D_{j-1}\right)$, the summation introduced in the previous sentence yields $1 /\left(k_{1} k_{2} \ldots k_{n}\right)$. Hence, by the first part of Theorem 2 , the proof is complete.

We record a special case of Theorem $2 \mathrm{a}$ as

Theorem 3. The integer parts $V_{j}$ of the ratios $D_{j} / H_{j-1}\left(D_{j-1}\right)$ are stochastically independent with distribution

$$
P\left(V_{j}=k\right)=\frac{1}{k(k+1)}, \quad k \geq 1 \text { and } j \geq 1,
$$

where $V_{1}$ is just $D_{1}$.

Theorem 3 is a very powerful tool for analyzing the growth rate of the sequence $D_{n}=D_{n}(x)$ when we seek the growth rate valid for almost all $x$. Note also that for the alternating Lüroth expansions, $H_{n}(j)=1$ for all $n$ and $j$, so $V_{n}=D_{n}$ in this case. Therefore, the subsequent statements on the sequence $V_{n}$ reestablish the results of Kalpazidou et al. [6] on the Lüroth case.

Set $R_{1}=D_{1}$, and for $n \geq 2$,

$$
R_{n}=D_{n} / H_{n-1}\left(D_{n-1}\right) .
$$

By definition, $V_{n}$ and $R_{n}$ differ by at most one, hence several statements on the sequence $V_{n}$ immediately transform to similar statements on the sequence $R_{n}$. We make use of this possibility in what follows, without repeatedly referring to this relation between $V_{n}$ and $R_{n}$.

3. Asymptotic results on $V_{n}$ and $R_{n}$. Because the expected value of $V_{n}$ is infinity, we have from probability theory

$$
\lim \frac{1}{n}\left(V_{1}+\ldots+V_{n}\right)=\infty \quad \text { for almost all } x(n \rightarrow \infty)
$$

and, for any $a>0$,

$$
\lim P\left(\left|\frac{1}{n \log n}\left(V_{1}+\ldots+V_{n}\right)-1\right|>a\right)=0 \quad(n \rightarrow \infty),
$$

which limit result cannot be extended to a limit for almost all $x$ (see Galambos $[3$, p. 63]). 
However, if one turns to logarithm, the strong law of large numbers applies and one gets

$$
\lim \left(\log V_{1}+\ldots+\log V_{n}\right) / n=E>0 \quad \text { finite } \quad \text { as } n \rightarrow \infty .
$$

All three limits above remain valid for the sequence $R_{j}$ in the place of $V_{j}$, $1 \leq j \leq n$.

When one analyzes the speed of convergence at (7), as $n \rightarrow \infty$, one needs an estimate on the product of $a_{j}\left(D_{j}\right) / b_{j}\left(D_{j}\right), 1 \leq j \leq n$. We turn to logarithm. By the definitions at (10) and (16),

$$
\begin{aligned}
\log \frac{a_{j}\left(D_{j}\right)}{b_{j}\left(D_{j}\right)} & =\log H_{j}\left(D_{j}\right)-\log D_{j}-\log \left(D_{j}+1\right) \\
& =-\log R_{j+1}+\log D_{j+1}-\log D_{j}-\log \left(D_{j}+1\right) .
\end{aligned}
$$

Upon summing the extreme sides above over $j$, from 1 to $n$, we get

$$
\sum_{j=1}^{n} \log \frac{a_{j}\left(D_{j}\right)}{b_{j}\left(D_{j}\right)}=\log D_{n+1}-\sum_{j=2}^{n+1} \log R_{j}-\log D_{1}-\sum_{j=1}^{n} \log \left(D_{j+1}+1\right) .
$$

The sum of $\log R_{j}$, when divided by $n$, converges to a finite number $A>0$ as $n \rightarrow \infty$ (see (A3)). So far we ignored $x_{n+1}$ in the error term at (7) (the last term of (7)). However, by (5), $1 / 2 \leq x_{n+1} D_{n+1} \leq 1$, whose logarithm is bounded. Hence, if $N(n)$ is a sequence of numbers tending to infinity faster than $n$, we have

THEOREM 4. If $N(n)$ goes to infinity faster than $n$, for the error term at (7) we have

$$
\frac{1}{N(n)}\left(\log x_{n+1}+\sum_{j=1}^{n} \log \frac{a_{j}\left(D_{j}\right)}{b_{j}\left(D_{j}\right)}\right)=-\frac{1}{N(n)} \sum_{j=1}^{n} \log \left(D_{j}+1\right) .
$$

To every classical expansion with positive terms such as that of Engel, Sylvester, Lüroth, Cantor and others (see Galambos [2, pp. 14-19] for a list) there is a corresponding alternating series expansion. For each of these, except for the case of Lüroth, $N(n)$ is indeed of a larger magnitude than $n$ when one seeks a finite nonzero limit on the right hand side of Theorem 4; consequently, Theorem 4 applies to all of the classical cases. For the exceptional case of Lüroth, the limits (A1)-(A3) correspond to Theorem 4. Since the basic distributional properties of the alternating series are similar to those in the case of expansions with positive terms, we refer to $[2$, pp. 106-109] for the choice of $N(n)$ for the classical cases.

4. The real role of the fundamental inequality (9). The fundamental inequality (9) is a necessary and sufficient condition for obtaining the digits $D_{j}$ at (7) by the expansion (5) and (6). In particular, there are numbers $x$ for which equality holds at (9) infinitely many times. 
However, if we allow a rule to apply for all $x$ except on a set of measure zero, then a significant change occurs at (9). We easily deduce from Theorem 3 that the set of $x$ for which

$$
D_{n+1} \geq 2 H_{n}\left(D_{n}\right)
$$

for all large $n$ is of measure zero because it would imply that, for such $x$ 's, $V_{j}=1$ would occur only a finite number of times, while Theorem 3 implies that $V_{j}$ should be one in about $50 \%$ of the cases (by one more application of the strong law of large numbers). What is then the real magnitude of growth for $D_{n}$ in the light of (9) and (18)? In one direction we get the following rule from the Borel-Cantelli lemmas.

TheOREM 5. The inequalities $D_{n+1} \geq k_{n} H_{n}\left(D_{n}\right)$ for arbitrary real numbers $k_{n}>1$ occur infinitely often either with probability one or with probability zero. It is of measure zero if, and only if, the sum of $1 / k_{n}$ over $n$ is finite.

In the opposite direction we extend a recent result of Lee [7] who proved that, for the newly introduced Daróczy-Kátai-Birthday (DKB) expansions (see Galambos [4]), one can get very close to the opposite inequality at (18). The DKB expansion of real numbers in an Oppenheim expansion with $h_{n}(j)=j^{2}(j-1)$. Its modification to alternating DKB expansion is the one obtained by the algorithm (5) and (6) with

$$
H_{n}(j)=j^{2}(j+1) .
$$

This is a cubic equation. We shall deal with expansions whose $H_{n}(j)$ is a polynomial of degree $t \geq 3$ with leading coefficient one. The cases $t=1$ or 2 have similar growth rates to those established below but their proof deviates somewhat from the one that follows. We therefore do not deal with those cases here.

Theorem 6. Let $H_{n}(j)=H(j)$ in (10) be a polynomial of degree $t \geq 3$ with leading coefficient one and assume that it is the same function for all $n$. Then, for almost all $0<x \leq 1$,

$$
D_{n+1} \geq D_{n}^{t}+D_{n}^{t} /\left(\log D_{n}\right)^{c} \quad \text { for all } n \geq n_{0}(x),
$$

where $c>0$ is an arbitrary constant.

Proof. Let $u_{j}, 1 \leq j \leq n$, be integers which are possible values of the $D_{j}$, i.e. for which the inequalities (9) hold. We shall refer to such values as realizable sequences (by the algorithm (5) and (6)). We fix $n$ and $u_{n}$, and estimate

$$
P\left(D_{n+1}<D_{n}^{t}+D_{n}^{t} /\left(\log D_{n}\right)^{c}\right) .
$$

The value in (21) is the sum over all realizable values $u_{j}, 1 \leq j \leq n+1$, where $u_{n+1}$ is further limited by (21). For a fixed set of $u_{j}$, Theorem 1 
applies. Next, we observe that

$$
\frac{a_{j}\left(u_{j}\right)}{b_{j}\left(u_{j}\right)}=\frac{H\left(u_{j}\right)}{j(j+1)},
$$

which, by assumption, is $j^{t-2}+O\left(j^{t-3}\right)$, with constant in $O($ ) not dependent on $j$. We do summation over a single $u_{j}, 1 \leq j \leq n-1$, starting with $u_{1}$. This way, the summands will be $u_{j}^{t-2}$ (with the appropriate error term that we shall take care of soon), and in view of $(9), u_{j}$ is limited by $u_{j+1}^{1 / t}$. We increase these sums if we sum over all integers for $u_{j}$ not exceeding $u_{j+1}^{1 / t}$, getting the bounds in the $j$ th summation

$$
\left(u_{j+1}\right)^{e(j)+1} /(e(j)+1)
$$

where the exponents $e(j)$ are defined by the recursive relation $e(1)=t-2$, and $e(j+1)=(e(j)+1) / t+t-2$. Upon solving this difference equation we get $e(j)=t-1-t^{-j+1}$. Finally, upon observing that $u_{n+1}$ satisfies both (9) and (21), summation over these values yields the estimate (recall (11) with $s=n+1)$

$$
1 /\left(u_{n+1}\left(u_{n+1}+1\right)\right) \leq u_{n}^{-2 t}\left(1+1 / u_{n}^{t}\right)
$$

multiplied by the number of terms between (9) and (21) which equals $u_{n}^{t} /\left(\log u_{n}\right)^{c}$.

When we collect the above terms for fixed $u_{n}$ we obtain on (21) the upper estimate

$$
\prod_{j=1}^{n-1}(e(j)+1)^{-1} \sum \frac{u_{n}^{e(n)}}{u_{n}^{t}\left(\log u_{n}\right)^{c}}
$$

where the summation is over all possible values of $u_{n}$. Now, since $u_{n}$ is larger than the values generated by (9) with equality for every $n$, the smallest value of $u_{n}$ satisfies $\log \left(u_{n}\right)>g t^{n}$ with some constant $g>0$ (see Lee [7], whose argument easily extends from $t=3$ to any $t \geq 3$ ). From this fact one easily sees that the sum above is finite. By the Borel-Cantelli lemma, the inequality in (21) fails for all large $n$ with probability one, i.e. (20) applies. The theorem is established.

Since the argument above applies in the case of expansions with positive terms as well, our result extends that of Lee [7], whose result is for $t=3$ (the DKB expansions), and his result is not for arbitrary $c>0$.

\section{References}

[1] J. Galambos, The ergodic properties of the denominators in the Oppenheim expansion of real numbers into infinite series of rationals, Quart. J. Math. Oxford Ser. (2) 21 (1970), 177-191. 
[2] J. Galambos, Representations of Real Numbers by Infinite Series, Lecture Notes in Math. 502, Springer, 1976.

[3] —, Advanced Probability Theory, 2nd ed., Marcel Dekker, New York, 1995.

[4] - Further metric results on series expansions, Publ. Math. Debrecen 52 (1998), 377-384.

[5] K.-H. Indlekofer, A. Knopfmacher and J. Knopfmacher, Alternating Balkema-Oppenheim expansions of real numbers, Bull. Soc. Math. Belg. 44 (1992), 17-28.

[6] S. Kalpazidou, A. Knopfmacher and J. Knopfmacher, Lüroth-type alternating series representations for real numbers, Acta Arith. 55 (1990), 311-322.

[7] M.-Y. Lee, On the Daróczy-Kátai-Birthday expansion, Publ. Math. Debrecen 58 (2001), 79-81.

[8] A. Oppenheim, The representation of real numbers by infinite series of rationals, Acta Arith. 21 (1972), 391-398.

[9] J. O. Shallit, Metric theory of Pierce expansions, Fibonacci Quart. 24 (1986), 22-40.

Department of Mathematics

Temple University, TU 038-16

Philadelphia, PA 19122, U.S.A.

E-mail: janos@math.temple.edu

Department of Mathematics

Dankook University

Seoul, South Korea

E-mail: leemy@dku.edu
Department of Computer Algebra Eötvös Loránd University H-1518 Budapest, Pf. 32, Hungary E-mail: katai@compalg.inf.elte.hu

Received on 3.4.2002

and in revised form on 2.10.2002 